\title{
Classifying Dynamics Events Using Neural Network and Wavelets for Current Power Systems
}

\author{
Yingli Shu ${ }^{1, a}$ \\ ${ }^{1}$ Changchun Institute Of Technology, Changchun, Jilin, China, 130021 \\ a shuyingli111@126.com
}

Keywords: Dynamics Events; Power System; Neural Network; Wavelet Analysis

\begin{abstract}
In this paper, we propose a novel methodology that classify the power system dynamics events patterns using neural network and wavelet through studying one single variable at a network bus. DWT allows the identification of components of the LFEO (low-frequency electromechanical oscillations), their frequencies, and magnitudes. Following the determination of the energy components' share of the studied signal using Parseval's theory and discrete wavelet transform, we get the input data. A total of five classes of disturbances, three different wavelet functions, and two different variables are tested. The experimental results emostrates that our methodology could classify different power disturbance types efficiently.
\end{abstract}

\section{Introduction}

A power system is a complex, dynamic system, composed of a large number of interrelated elements, where some elements are merely the system's components while others affect the whole system [1]. Several large-scale blackouts of power systems have occurred worldwide over the past 10 years. One of the largest in European history was the collapse of the electricity power system in Italy, on 28 September 2003, resulting in the loss of electricity for 57 million people. An analysis of these collapses indicates that the most common causes are the chain propagation of the initial disturbance with high intensity and some failures in operation and power system planning [2]. The modern approach to power system monitoring, operation, and control involves the fast and accurate identification and localization of the disturbance. Accurate and fast automatic identification of the disturbance type and location can help the system operator with the proper initiation of corrective actions that would eliminate or reduce the impact of disturbances on the system. Generally, the objectives of disturbance identification are to detect the onset in time of disturbance, classify the type of disturbance, estimate the disturbance intensity and damping, and estimate the end time of the disturbance and the type and location of the initial event. A new approach for the classification of power system dynamic events based on WT and a neural network is proposed in this paper. Discrete wavelet transform with multi-resolution analysis and research is applied to decompose signals of electromechanical transient oscillation in a frequency range of up to $5 \mathrm{~Hz}$. DWT with MRA allows the identifications of power system LFEOs and the determination of their frequencies and magnitudes. Following the identification of LFEOs in the signal that are coming out of the DWT, Parseval's theorem is used to determine the energy share of the signal DWT decomposition levels, whose values represent the input to the NN used for classification. Five classes of power system disturbances, which can represent higher intensity disturbances, are analyzed as follows: loss of generation units, line outage, 3-phase short circuit (TPSC), TPSC with a line outage, and TPSC with a line outage and unsuccessful reclosing. Performances of different wavelet functions are tested.

\section{Our Proposed Methodology and Related Work}

Background Analysis. The basic idea of the proposed methodology is based on the identification component of the LFEOs of the power system and determining their effects on the observed variables 
using DWT. The LFEOs of a power system are in the range of up to $5 \mathrm{~Hz}$ [3]. The block diagram for the power system events classification proposed in this paper is presented in Figure 1.



Figure.1 The Block Diagram

Our Proposed Methodology. The first step is the selection of analyzed variables and the definition of the measured signal sampling frequency. Considering the time and frequency range of electromechanical transients of power systems, as referred to in [2], it is common for them to be observed on a time scale of a few seconds and in a frequency range of up to $5 \mathrm{~Hz}$. Selecting a signal sampling time of $0.1 \mathrm{~s}$ or sampling frequency of $10 \mathrm{~Hz}$, it will include all frequencies in the range of up to $5 \mathrm{~Hz}$ and thus will eliminate electromagnetic transients from the signal that will be signiifcantly reduced by the number of signal samples. The second step is the DWT analysis of the selected signal. The dynamic response of a power system following a disturbance is characterized by significant uctuations of the angles between the generators. The third step is the classification of the disturbances by the NN. There are several types of NNs that can be used in the classification process. In this study, a feed-forward NN (FFNN) is used. Generally, for ANNs, it is common that the accumulated knowledge during training can be utilized properly for some subsequent events. The FFNN consists of an input layer, output layer, and hidden layers. The connections have multiplying weights associated with them. Specifically, determining the number of neurons and hidden layers is a common problem in the NN method implementation. The process of determining the parameters of the NN is called the training process, during which the sets of input and output data are associated by proper adjustment of the weights in the network and the sum of the squared error function is minimized. The training process involves a specified learning rule. More details on the types and architectures of NNs can be found in the literature [4].

\section{Experimental Result and Analysis}

The simulation and analysis presented in this paper were performed using the New England (NE) 39-bus test system presented in Figure 2. The NE 39-bus test system consists of 10 generators connected at buses 30 to 39, where bus 31 is a slack bus. All of the generators are equipped with identical automatic voltage regulators (AVRs) and turbine governors (TGs). The loads are modeled as voltage-dependent loads. The load and line data of the test system come from [5], and the AVR and TG parameters come from [5]. The performance of the proposed approach was evaluated by the Power System Analysis Toolbox [5] and Wavelet Toolbox .

In every simulated power system disturbance case, the voltage angle and voltage magnitude signals at bus 16 are observed in a 10-s simulation time frame. Bus 16 is chosen randomly as one of the central buses. The start of the disturbance in each simulated case is at $0.1 \mathrm{~s}$ and all of the voltage angle signals are presented in relation to bus 39. Just for illustration, when speaking about the identification of the LFEO and the comparison of the different methodologies, we analyze 1 simulated TPSC disturbance case on bus 7, starting at $5.1 \mathrm{~s}$ and ending at $5.2 \mathrm{~s}$. The total simulation time is 30s, as taken just for illustration. After the disturbance simulation, oscillations are observed 
throughout the system. Figure 3 shows the voltage angle oscillations on bus 16 after the TPSC on bus 7. It is clear from the signal observed in Figure 3 during the TPSC interval that a smaller transient is present, but after the disturbance clearance, the system continues to oscillate. It is obvious that the oscillations are damped, but some components might not be damped, even if it cannot be seen from the observed signal[6]. In a case where we observe the voltage magnitude, the transient or 'sag', the actual voltage magnitude decrease would be substantially higher due to the nature of the QV process during the short circuit.

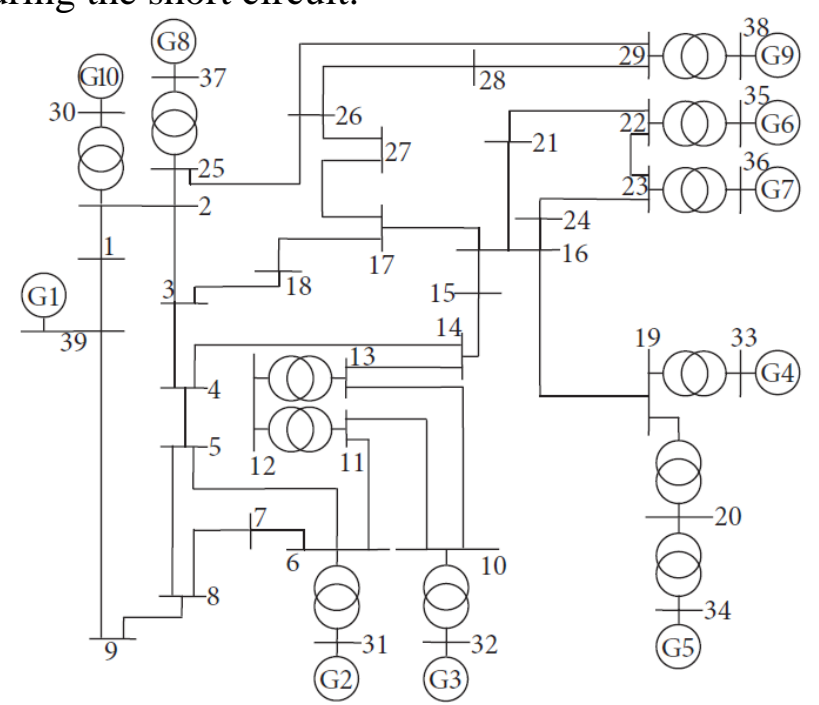

Figure.2 The NE 39-bus Test System

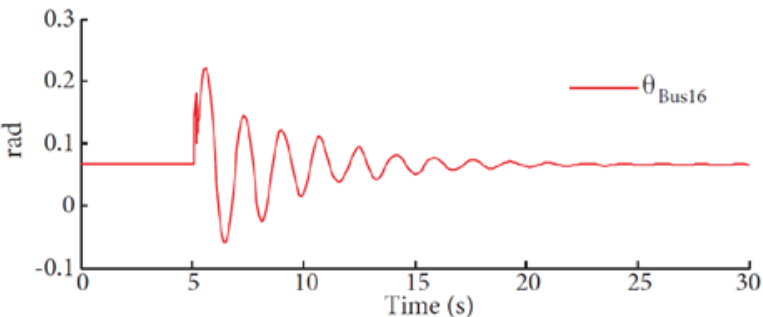

Figure.3 Voltage angle oscillations

The results of the analysis of the signal from Figure 3 using the Prony approach are shown in Figure 4 and also indicate the dominant frequency of $0.6 \mathrm{~Hz}$. This approach would not only get the characteristic frequencies, but also their damping. By modal form signal representation, one can _nd the corresponding amplitudes and also evaluate the energy contribution of the signal components. However, this method loses the information about the beginning of a power system disturbance. WT provides an excellent time-frequency analysis and the ability of local signal analysis. It also enables the usage of a longer time interval for the 'capture' of information about low-frequency components, and shorter time intervals for information about high-frequency components. The wavelet power spectrum and global wavelet spectrum of the signal results from Figure 3 are presented in Figure 5. Moreover, the dominant frequency component is $0.6 \mathrm{~Hz}$, but in addition to information about the low-frequency components, this approach enables us to visualize the beginning of an underlying disturbance that presents important information for power system operators.

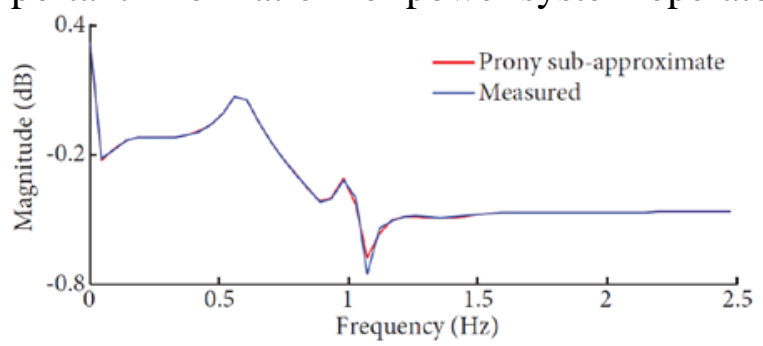

Figure.4 Prony approach results of the signal from Figure 3.

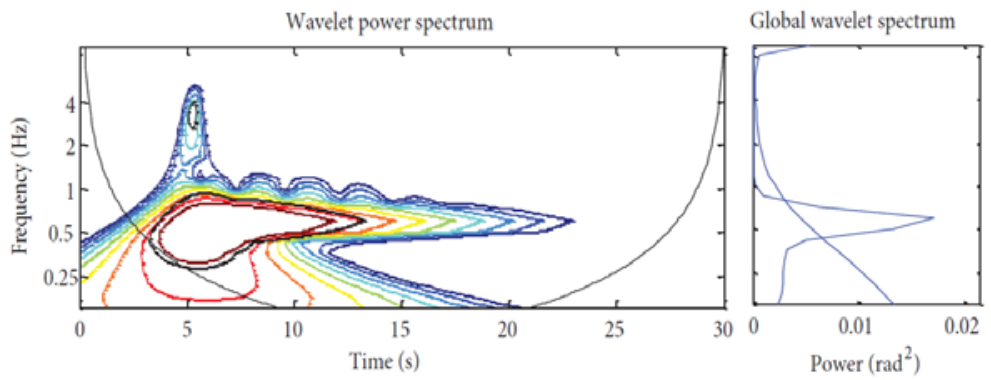

Figure.5 Wavelet Power Spectrum and Global Wavelet Spectrum Results 
Better results in terms of the classi_cation results should be looked at by more careful selection of each element of the training set, using the appropriate tools for their _ltering, in order to avoid over-fitting and an eventual increase of the variance results during the network testing. A technique of factor analysis (principal component analysis) could be applied in this or a similar analysis in order to possibly reduce the number of input variables, with no signi_cant reduction in the total variability in the data. Although the FFNN is commonly used in the literature because of its simplicity setting, easier automation of the network selection, and its application, it is possible to _nd a successful application of a large number of other types of classifiers. In the case of the FFNN, the optimal choice of hidden layers and the number of neurons per layer might have an important role in the process of classi_cation. In the case of over-fitting, it may happen that the training is done very successfully, but for a variety of architectures of the FFNN, one gets di_erent variances during the network testing, thus making the classification inconvenient. Radial basis function, cascade NN, and Kohenen NN are some of the NNs that can be applied in the classification process. Since there are no general rules for the election of the classifier, for each specific problem, and even for that analyzed in this article, the specific analysis with different types of classifiers would advise the possible choice of one classi_er that gives the best results.

\section{Summary and Conclusion}

In this paper, using DWT and a NN, the possibility of the classification of high-intensity disturbances in a power system was analyzed by observing one variable at a single network bus. The proposed approach is based on the identification of the dominant low-frequency components of the analyzed signal and determination of their participation with energy in the signal by DWT. From the performed simulations, analyses, and classifications, the following conclusions can be drawn.

The LFEO will arise in the system after the disturbance. The magnitude of these modes will, among other things, depend on the type of disturbance. Different wavelet functions of the DWT of the analyzed signals give different output signals from the filters, which affect the energy distribution for different frequency bands. However, it is obvious that the selection of the wavelet function does not affect the classification results. Furthermore, an important aspect of the proposed approach is the selection of the variables. In the future research, we would plan to improve the accuracy of the classification result for the input signal. Moreover, we plan to use novel machine learning technology method to improve our neural network, such as deep learning and mathematical optimization[7,8].

\section{References}

[1] P.M. Anderson, Power System Control and Stability, 2nd ed., New York, Wiley-IEEE Press

[2] P. Kundur, Power System Stability and Control, New York, McGraw-Hill.

[3] M. Ili_c, J. Zaborszky, Dynamics and Control of Large Electric Power Systems, New York.

[4] B. Pal, B. Chaudhuri, Robust Control in Power Systems. New York, Springer.

[5] M. Vetterli, J. Kovacevic, Wavelets and Subband Coding, New York, Prentice-Hall.

[6] S. Mallat, A Wavelet Tour of Signal Processing, San Diego, CA, USA, Academic Press

[7] Chen, Guang-Sheng, et al. "Existence of three solutions for a nonlocal elliptic system of-Kirchhoff type." Boundary Value Problems 2013.1 (2013): 1-9.

[8] Dong, Chao, et al. "Learning a deep convolutional network for image super-resolution." Computer Vision-ECCV 2014. Springer International Publishing, 2014. 184-199. 